\title{
Potentiation of Bio Repositories In Personalized Medicine: Tumor Cells Establishment
}

\author{
Cheng- Li*, Ying Chu \\ Department of Dermatology, Taipei Veterans General Hospital, Taiwan.
}

\begin{abstract}
"Corresponding Author : Cheng-Yuan Li, ${ }^{1}$ Department of Dermatology, Taipei Veterans General Hospital, Taiwan , E-mail: chengli@yahoo.com Received date: August 20,2017;Accepted date : September 12,2017; Published date: September 19,2017.

Citation for this Article: Cheng-Yuan Li, Ying Chu, Potentiation of Bio Repositories In Personalized Medicine: Tumor Cells Establishment , J Cancer
\end{abstract} Research and Cellular Therapeutics, Doi: 10.31579/2640-1053/003

Copyright : (c) 2017 Cheng-Yuan Li . This is an open-access article distributed under the terms of The Creative Commons Attribution License, which permits unrestricted use, distribution, and reproduction in any medium, provided the original author and source are credited.

\begin{abstract}
The introduction of three-dimensional (3D) tumor cultures has revolutionized anticancer drug research as these cultures allow for the study of drug resistance mechanisms that cannot be explored in traditional two dimensional (2D) monolayer cultures. Discoveries in the 3D tumor culture field suggest that individualized drug sensitivity testing of solid tumor specimens through the establishment and use of 3D tumor cell cultures following tissue collection will become a routine service offered by modern tissue repositories as they expand from their traditional research role to active participation in personalized medicine. Unfortunately, most information related to 3D tumor cultures comes from studies using established tumor cell lines rather than primary tumor cultures. However, accumulation of genetic aberrations in cancer cell lines occurs with increasing number of passages severely limiting their usefulness for personalized medicine. There is only very limited information available concerning technologies and standard operating procedures for the efficient and routine isolation and processing of primary tumor cells for the establishment of 3D tumor cultures from solid tumor specimens. The purpose of this work was to review experimental data from the literature that may provide relevant information concerning the isolation and processing of primary tumor cells for the establishment of 3D tumor cultures. Information reviewed here may help bio repositories in the development and standardization of technologies and standard operating procedures related to the use of 3D tumor cultures.
\end{abstract}

\section{Introduction}

Accumulation of genetic aberrations in cancer cell lines occurs with increasing number of passages severely limiting their usefulness for personalized medicine [1,2]. In contrast, the critical importance of technologies related to the derivation, short term culture, and testing of primary tumor cells from solid tumors is increasingly recognized (for a recent review see 2). Short-term primary cultures of tumor cells derived from pieces of solid tumors have been used for predicting anticancer drug responses [3,4]. Observations suggest that individualized drug sensitivity testing of solid tumors through the isolation and culturing primary tumor cells in $3 \mathrm{D}$ may soon become routine and modern tissue repositories will need to be ready to support these activities as they expand from their traditional research role to active participation in personalized medicine [5].

Cancer cells grown in 3D cultures in a polymeric ECM closely mimic the biology of tumor development in vivo and numerous studies indicate that $3 \mathrm{D}$ cultures are superior to traditional 2D monolayer cultures for studies of key cellular behaviors like differentiation, proliferation, invasion and apoptosis [6-8]. Cancer cells grown in 3D culture are more resistant to chemotherapeutic agents, radiation and oncolytic virotherapy than cells in $2 \mathrm{D}$ culture and 3D tumor cell cultures are useful for preclinical evaluation of the cytotoxic effect of anticancer agents [6, 9-16].

Unfortunately, most information related to 3D tumor cultures comes from studies using established tumor cell lines rather than primary tumor cultures and there is only very limited information available concerning technologies and SOPs for the efficient and routine isolation and processing of primary tumor cells for the establishment of $3 \mathrm{D}$ tumor cultures from solid tumor specimens.
The purpose of this work was to review experimental data from the literature that may provide relevant information concerning the isolation and processing of primary tumor cells for the establishment of 3D tumor cultures.

\section{Materials and Methods}

A review of the English language literature related to the isolation and processing of primary tumor cells for the establishment of $3 \mathrm{D}$ tumor cultures from solid tumor specimens was performed.

\section{Results}

Only a limited number of reports are available related to the isolation and processing of primary tumor cells for the establishment of $3 \mathrm{~d}$ tumor cultures from solid tumor specimens. However, these relate to colorectal, lung and brain tumors indicating that isolation and growth of primary tumors cells in 3D cultures is possible for a variety of solid tumors. The protocols have an initial step of mechanical mincing of tissues followed by at least some disruption of the original extracellular matrix by enzymatic dissociation. Isolated tumor cells or fragments can be grown and analyzed in a wide variety of $3 \mathrm{D}$ culture systems. Four prominent examples are reviewed below.

\section{Example 1. Kondo J et al. 2011 [17].}

Kondo et al found that retaining cell-cell contact enables preparation and culture of spheroids composed of pure primary cancer cells from colorectal cancer specimens that retain the characteristics of the parental tumors, grow in vitro in $3 \mathrm{D}$ cultures and could be used for personalized diagnostic applications, including chemosensitivity assays. 
To successfully maintain the cell-cell contact, they used Liberase DH (Roche Diagnostics) as a blend of digestion enzymes. They optimized the enzyme concentration and the digestion time to avoid overdigestion of colorectal tissues. Cancer specimens from colorectal cancer patients were mechanically and enzymatically digested and separated into two fractions using a cell strainer: the organoid fraction (ORG), which was retained in a 40-?m strainer, and the flow-through fraction (FT), which passed through the strainer. The FT fraction mainly contained single cells and many of the cells with epithelial markers were dead. In contrast, the ORG contained irregular sheet- or tube-like structures, which were termed organoids. After overnight culture, the organoids became spherical and bright with a smooth surface and were termed cancer tissue-originated spheroids (CTOSs). During formation, the CTOSs were draped with cellular debris that easily detached with pipetting. The diameter of the CTOSs was?40$500 ? \mathrm{~m}$, depending on the cell strainer size. A CTOS with a diameter of $100 ? \mathrm{~m}$ consisted of ?100 cells. The authors were able to prepare CTOSs in most of the colorectal cancer specimens regardless of the disease stage and histology. CTOSs were highly viable with only a few dead cells on the outside. CTOSs could be cultured and expanded in vitro. Extracellular matrix was critical for favorable growth of CTOSs. Excellent proliferation was accomplished with the use of a 3D type I collagen-based culture system and serum-free medium designed for embryonic stem cells. The success rate for growth was $73.5 \%$. CTOSs were composed of highly purified and viable cancer cells, that retained the characteristics of the parental tumors, grew in vitro and could be used for personalized diagnostic applications, including chemosensitivity assays.

\section{Example 2. Endo H. et al. 2013 [18].}

This study demonstrated the usefulness of cancer tissue-originated spheroid (CTOS) method for the primary culture of lung cancer cells. Surgical specimens and pleural effusion samples from lung cancer patients were minced with a scalpel into approximately one square $\mathrm{mm}$ pieces, and washed with Hank's balanced salt solution. Specimens were then digested in DMEM supplemented with Liberase $\mathrm{DH}$, at $37^{\circ} \mathrm{C}$ for 1 to 2 hours with gentle stirring by a magnetic bar. Digested tissue suspensions were passed through $500-? \mathrm{~m}$ and $250-? \mathrm{~m}$ metal mesh filters to remove large masses of undigested fragments. Suspensions were further filtered through 100-?m and 40-?m cell strainers. Fragments on the cell strainer and cells in the flow-through fractions were collected separately, and were each washed with HBSS and cultured in StemPro hESC medium in a nontreated dish. Pleural effusions were transferred to $50-\mathrm{ml}$ tubes and centrifuged at $200 \mathrm{~g}$. Pellets were resuspended in HBSS, filtered through 40-?m cell strainers, and collected and cultured in the same manner as surgical specimens. CTOSs were embedded in Matrigel GFR and cultured in 100 ?1 of basal medium containing growth factors (NRG1, LongIGF1, bFGF , Activin A, or EGF). Basal medium consisted of DMEM/F12, 2\% BSA fraction V, nonessential amino acids, penicillin, streptomycin, ascorbic acid, human transferrin, ?-mercaptoethanol, and trace elements. Lung carcinoma CTOSs grew in vitro and could be used for personalized chemosensitivity assays.

\section{Example 3. Jiquet Jiglaire et al. 2014 [19].}

Glioblastoma tumor samples were automatically sectioned using a tissue-chopper. Cell suspensions were obtained after enzymatic dissociation with both $5 \mathrm{mg} / \mathrm{mL}$ of trypsin and $200 \mathrm{U} / \mathrm{mL}$ of DNAse for $10 \mathrm{~min}$ at $37 \mathrm{1C}$. Cell suspension filtration was done on a $0.40 \mathrm{~mm}$ filter and suspension was centrifuged at $1300 \mathrm{rpm}$ for $5 \mathrm{~min}$. Cells were resuspended in PBS and were grown in a commercially available 3D hydrogel (ExtracelTM, Tebu- bio, LePerray-en Yvelines,France).

\section{Example 4. Xu Z et al. 2013 [16].}

Cells from fresh lung cancer tissues were cultured and tested in 3D using a microfluidic chip-based, three-dimensional (3D) co-culture drug sensitivity test platform. Immediately after removal, lung cancer specimens were immersed in culture medium and kept cold while being transported to the lab.
The necrotic areas, fatty tissue, blood clots, and connective tissue were removed. The tumors were finely minced then digested with collagenase I $(0.3 \mathrm{mg} / \mathrm{mL})$ for $2 \mathrm{~h}$ at $37 \mathrm{C}$. The culture medium was collected and centrifuged (200 g, $5 \mathrm{~min}$ ). The sediments were prepared as single cell suspensions in serum-free DMEM and separated into different fractions using Percoll discontinuous gradients centrifugation (400 g, $20 \mathrm{~min} ; 30 \%$ and $70 \%$ Percoll). The cells at the interface of $30 \%$ and $70 \%$ Percoll were collected and washed twice with PBS to remove the gradient medium and obtain the cancer cells. Then, the primary celleBME mixtures were introduced into the chambers of a microfluidic chip-based, threedimensional (3D) co-culture system.

\section{Discussion}

Accumulation of genetic aberrations in cancer cell lines occurs with increasing number of passages severely limiting their usefulness for personalized medicine [1,2]. Research reviewed here indicates that isolation and culturing of primary tumor cells in 3D is possible and it is likely that primary 3D tumor cultures will soon be routinely used for individualized solid tumor testing. Modern tissue repositories will need to be ready to support these activities as they expand from their traditional research role to active participation in personalized medicine.

Published protocols for the isolation and culturing of primary tumor cells in 3D consist of an initial step of mincing of solid tumor specimens followed by at least some disruption of the original extracellular matrix by enzymatic dissociation. Isolated tumor cells or fragments can be grown and analyzed in a wide variety of 3D culture systems. Research indicates that methods for isolation and processing of primary tumor cells for the establishment of 3D tumor cultures need to be tumor type specific. As more research findings become available in this clinically highly relevant field, it will be important to standardize tissue and tumor-specific protocols related to the establishment of primary 3D tumor cell cultures. An introduction of 3D primary tumor cultures in routine bio repository activities will also require an adjustment of standard operating procedures, consent procedures and institutional review board (IRB) protocols to include routine isolation of primary tumor cells at the time of tumor tissue collection.

\section{References}

1. Gillet JP, Calcagno AM, Varma S, Marino M, Green LJ et al. (2011) Redefining the relevance of established cancer cell lines to the study of mechanisms of clinical anti-cancer drug resistance. PNAS USA.

2. Mitra A, Mishra L, Li S (2013) Technologies for deriving primary tumor cells for use in personalized cancer therapy. Trends Biotechnol 31: 347-354.

3. Brower SL, Fensterer JE, Bush JE (2008) The ChemoFx assay: an ex vivo chemosensitivity and resistance assay for predicting patient response to cancer chemotherapy. Methods Mol Biol 414: 57-78.

4. Jain KK (2009) Textbook of personalized medicine. Springer.

5. Valyi-Nagy K (2013) Three-dimensional tumor cell cultures and the role of tissue biorepositories in personalized medicine. J Clin Anat Pathol 1: 1-2.

6. Xu F, Burg KJ (2007) Three-dimensional polymeric systems for cancer cell studies. Cytotechnology 54: 135-143.

7. Page H, Flood P, Reynaud EG (2013) Three-dimensional cell cultures: current trends and beyond. Cell Tissue Res 352: 123-131.

8. Kimlin LC, Casagrande G, Virador VM (2013) In vitro threedimensional (3D) models in cancer research: an update. Mol Carcinog 52: 167-182.

9. Breslin S, O'Driscoll L (2013) Three-dimensional cell culture: the missing link to drug discovery. Drug Discov Today 18: 240-249.

10. Smalley KS , Lioni M, Herlyn M (2006) Life isn't flat: taking cancer biology to the next dimension. In Vitro Cell Dev Biol Anim 42: 242-247.

11. Valyi-Nagy K, Folberg R, Valyi-Nagy T, Maniotis A (2007) Susceptibility of uveal melanoma to herpes simplex virus type 1: the role of tumor invasiveness, the extracellular matrix and chromatin sequestration. Exp Eye Res 84: 991-1000. 
12. Valyi-Nagy K, Dosa S, Kovacs SK, Bacsa S, Voros A et al. (2010) Identification of virus resistant tumor cell subpopulations in three dimensional uveal melanoma cultures. Cancer Gene Ther 17: 223-234.

13. Valyi-Nagy K, Voros A, Gagyi E, Valyi-Nagy T (2011) Increased Resistance of Vasculogenic Mimicry-Forming Uveal Melanoma Cells against Cytotoxic Agents in ThreeDimensional Cultures, Research on Melanoma - A Glimpse into Current Directions and Future Trends, Mandi Murph (Ed.), InTech, Chapter 18, 377-392.

14. Valyi-Nagy K, Kormos B, Ali M, Shukla D, Valyi-Nagy T (2012) Stem cell marker CD271 is expressed by vasculogenic mimicry-forming uveal melanoma cells in three-dimensional cultures. Mol Vis 18: 588-592.

15. Voros A, Kormos B, Valyi-Nagy T, Valyi-Nagy K (2013) Increased resistance of breast, prostate and embryonic carcinoma cells against herpes simplex virus in threedimensional cultures. ISRN Oncol, Article ID 104913.
16. Xu Z, Gao Y, Hao Y, Li E, WangY et al. (2013) Application of a microfluidic chip-based 3D co-culture to test drug sensitivity for individualized treatment of lung cancer. Biomaterials 34 (2013) 4109-4117.

17. Kondo J, Endo H, Okuyama H, Osamu Ishikawa O, Iishi $\mathrm{H}$ et al. (2011) Retaining cell-cell contact enables preparation and culture of spheroids composed of pure primary cancer cells from colorectal cancer. Proc Natl Acad Sci U S A 108: 6235-6240.

18. Endo H, Okami J, Okuyama H, Kumagai T, Uchida J et al. (2013) Spheroid culture of primary lung cancer cells with neuregulin 1/HER3 pathway activation. J Thorac Oncol. 8: 131-139.

19. Jiquet Jiglaire C, Baeza-Kallee N, Denicolaï E, Barets D, Metellus P et al. (2014) Ex vivo cultures of glioblastoma in three-dimensional hydrogel maintain the original tumor growth behavior and are suitable for preclinical drug and radiation sensitivity screening. Exp Cell Res 321: 99-108. 